\title{
SYNTHESIS AND PROPERTIES OF CHALCONES BASED
} ON DEHYDROACETIC ACID

\section{Ya. Chernii ${ }^{*}$, I. M. Tretyakova ${ }^{1}$, N. M. Fedosova ${ }^{1}$, I. M. Denysenko ${ }^{1}$, Ya. M. Dovbii ${ }^{1}$, V. B. Kovalska ${ }^{1,2}$, S. V. Chernii ${ }^{1,2}$, V. I. Pekhnyo ${ }^{1}$, A. S. Starukhin ${ }^{3}$}

${ }^{1}$ V. I. Vernadskii Institute of General and Inorganic Chemistry NAS of Ukraine, Kyiv, Ukraine ${ }^{2}$ Institute of Molecular Biology and Genetics NAS of Ukraine, Kyiv, Ukraine

${ }^{3} B$. I. Stepanov Institute of Physics NAS of Belarus, Minsk, Belarus

*e-mail:v.chernii@gmail.com

The Knoevenagel condensation reaction between dehydracetic acid and aromatic aldehydes is described in this work. The reaction is carried out directly between dehydroacetic acid and aromatic aldehydes in the presence of organic bases. The optimal conditions for the Knoevenagel reaction based on dehydroacetic acid and various aldehydes were determined. Twenty-one chalcones with substituents of different nature were synthesized. The composition and structure of the obtained compounds were determined. All characteristic signals of chalcones are present in the ${ }^{1} \mathrm{H}$ NMR spectra of the obtained compounds registered in $\mathrm{CDCl}_{3}$ and DMSO- $\mathrm{d}_{6}$ : $\mathrm{OH}$ groups in the range of 18.7-16.5 ppm, $\mathrm{CH}$ proton $-6.3-5.9 \mathrm{ppm}$, and methyl group of the pyran cycle $2.3-2.2 \mathrm{ppm}$. The corresponding signals of methine protons and aryl substituents are also present in the spectra. The most sensitive to solvent changes is the $\mathrm{OH}$ proton bound by an intramolecular hydrogen bond to the carbonyl group of the pyran ring. Signals in DMSO are usually shifted by $0.1-1.0 \mathrm{ppm}$ in a stronger field compared to $\mathrm{CDCl}_{3}$ for dehydroacetic acid and chalcones based on it. $\mathrm{CH}$ proton signals are shifted by approximately $0.3 \mathrm{ppm}$ in a weaker field, and the signals of the protons of the methyl group are almost insensitive to the solvent. The optical properties of obtained compounds were investigated in $\mathrm{DMF}, \mathrm{MeOH}, \mathrm{MeCN}$. The synthesized chalcones absorb light in the visible range 330-490 nm with molar extinction coefficients of 3.5-4.5. The solvatochromic effects for most of them are weak - the position of the maximum changes by less than $10 \mathrm{~nm}$. The electron-donor substituents in the phenyl ring $\left(-\mathrm{NMe}_{2}\right.$ and $\left.-\mathrm{NEt}_{2}\right)$ shift the absorption maximum bathochromically by almost $100 \mathrm{~nm}$ compared to others in all investigated solvents.

Keywords: dehydroacetic acid, Knoevenagel condensation, chalcones. 
INTRODUCTION. Dehydroacetic acid (3-acetyl-4-hydroxy-6-methyl-2H-pyran-2-one, (DHA)) is a $\alpha$-pyrone derivative. Its structure was first established by Hale in 1911 [1]. Complexes of dehydroacetic acid with ions of aluminum, zinc, copper(II), beryllium, manganese (II), and other metals are described in the literature. DHA is proposed as a complexing agent in analytical chemistry for gravimetric determination of copper, aluminum, and beryllium [2]. The corresponding mixed ligand complexes are formed when DHA interacts with zirconium and hafnium phthalocyanines [3]. An annealed analog of dehydroacetic acid - 3-acetyl-4-hydroxycoumarin has been proposed for the determination of titanium(IV), cerium(IV), thorium(IV), uranium(VI), and iron(II) ions $[4,5]$. X-ray diffraction data obtained for dehydroacetic acid complexes with manganese(II) ( $\left.\mathrm{Mn}(\mathrm{DHA})_{2}\left(\mathrm{CH}_{3} \mathrm{OH}\right)_{2}\right)$, cadmium, and zinc $[6,7]$, confirm the formation of chelate complexes in which the metal atom is coordinated through 3-acetyl and 4-oxy groups of dehydroacetic acid.

DHA is also widely used in organic chemistry since it enters into dozens of reactions to form very diverse classes of substances [8] (Fig. 1).

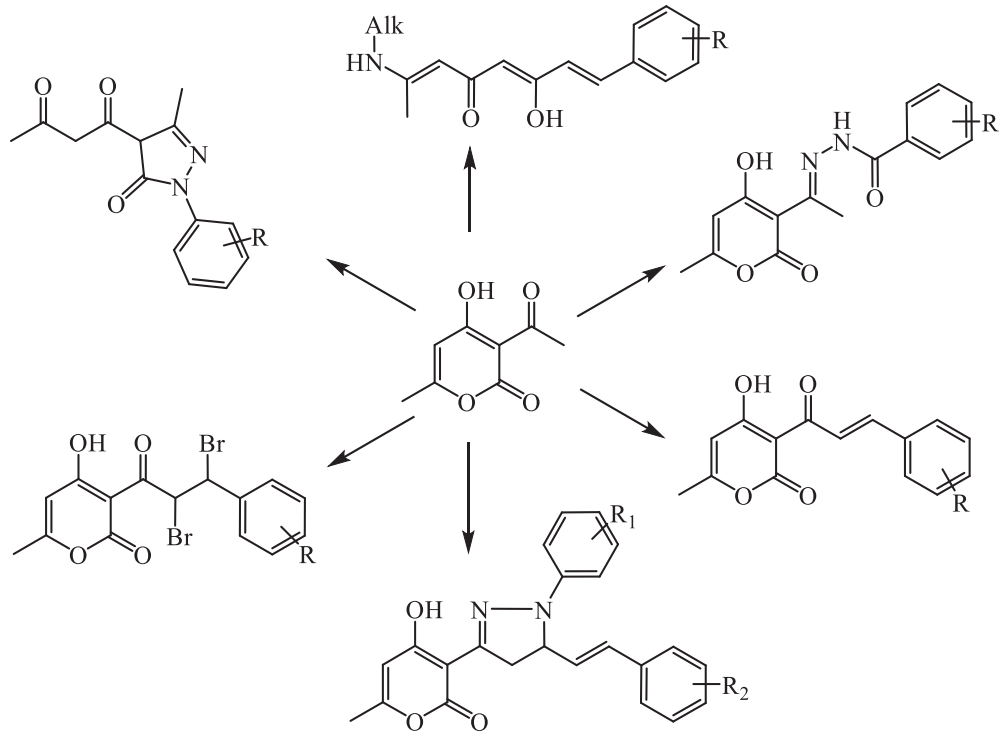

Fig. 1. Examples of dehydroacetic acid derivatives containing chelating centers.

Some of these compounds contain $\beta$-keto-enol or other fragments that may be promising to inorganic chemists as ligands. For example, the interaction of dehydroacetic acid with aromatic aldehydes by the Knoevenagel reaction produces the corresponding chalcones [9-14]. These are colored substances that can also form complexes with metals [15] and boron [16].
Metal complexes have also been widely studied not only with dehydroacetic acid [17-19] but also with its derivatives - Schiff bases [20-25] and other various $\mathrm{O}, \mathrm{N}, \mathrm{S}$ donor ligands [26-28]. For dehydroacetic acid and its derivatives, antimicrobial [15, 29], antitumor [30, 31], antiviral, in particular, anti-HIV activity $[16,32,33]$, etc., are widely studied. 
The interaction of chalcones with aliphatic amines opens the pyran cycle, preserving the chromophore fragment and forming the corresponding alkylamino- $\beta$-keto-enols. These compounds have found their application to monitor the amyloid fibril formation of proteins $[34,35]$ and as fluorescent probes for functional amyloid visualization in biofilm by confocal microscopy [36].

There are two main methods for preparing chalcones based on dehydroacetic acid by the Knoevenagel reaction (Fig. 2).
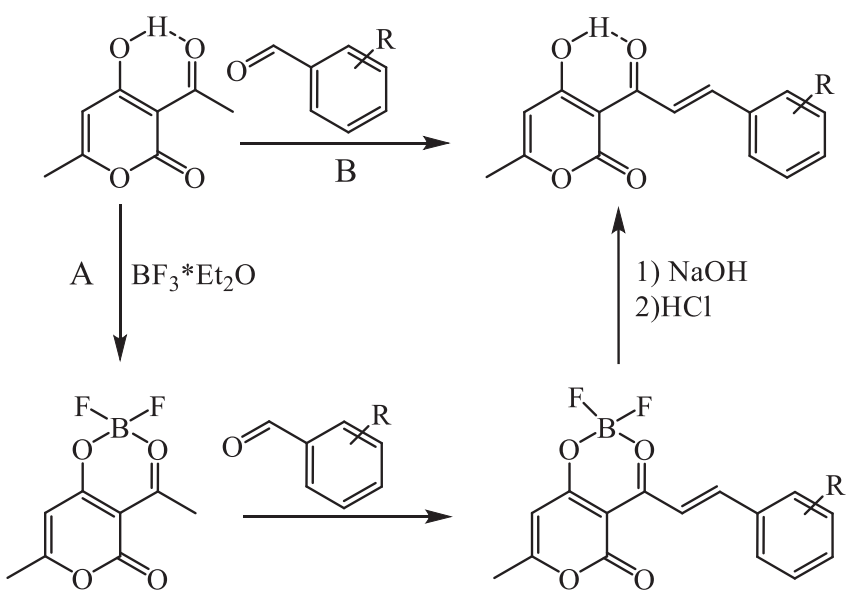

Fig. 2. Methods of obtaining chalcones - derivatives of dehydroacetic acid: activation of the methyl group by forming boron difluoride complex (A), the direct reaction between dehydroacetic acid and aromatic aldehydes (B).

According to the first method, the methyl group of dehydroacetic acid is activated by converting dehydroacetic acid into a boron difluoride complex [16], which reacts with aromatic aldehydes with the formation of the corresponding complex. In the next step, this compound is hydrolyzed with alkalis to the corresponding chalcone. According to the second method, the reaction is carried out directly between dehydroacetic acid and aromatic aldehydes [1] in the presence of organic bases, for example, piperidine.

We have obtained a wide range of chalcones with substituents of different natures in the aromatic nucleus (Fig. 3) and investigated their spectral properties.<smiles></smiles>
1) $\mathrm{R}=\mathrm{H}$
10) $\mathrm{R}=4-\mathrm{OMe}$
2) $\mathrm{R}=2-\mathrm{NO}_{2}$
11) $\mathrm{R}=4-\mathrm{OPr}$
3) $\mathrm{R}=3-\mathrm{NO}_{2}$
12) $\mathrm{R}=3-\mathrm{OMe}, 4-\mathrm{OH}$
4) $\mathrm{R}=4-\mathrm{NO}_{2}$
13) $\mathrm{R}=2,4-(\mathrm{OMe})_{2}$
5) $\mathrm{R}=2-\mathrm{OH}$
6) $\mathrm{R}=3-\mathrm{OH}$
14) $\mathrm{R}=3,5-(\mathrm{OMe})_{2}$
7) $\mathrm{R}=4-\mathrm{OH}$
15) $\mathrm{R}=3,4,5-(\mathrm{OMe})_{3}$
8) $\mathrm{R}=2-\mathrm{OMe}$
16) $\mathrm{R}=3,4-\mathrm{OCH}_{2} \mathrm{O}$
9) $\mathrm{R}=3-\mathrm{OMe}$
17) $\mathrm{R}=4-\mathrm{F}$
18) $\mathrm{R}=4-\mathrm{NMe}_{2}$
19) $\mathrm{R}=4-\mathrm{NEt}_{2}$

20)<smiles>Cc1cc(O)c(C(=O)/C=C/c2cccc3ccccc23)c(=O)o1</smiles>

21)<smiles></smiles>

Fig. 3. Molecular structures of obtained chalkones

\section{EXPERIMENT AND DISCUSSION OF} THE RESULTS. All characteristic signals of chalcones are present in the ${ }^{1} \mathrm{H}$ NMR spectra of the obtained compounds registered in $\mathrm{CDCl}_{3}$ and DMSO- $\mathrm{d}_{6}: \mathrm{OH}$ groups in the range of 
18.7-16.5 ppm, $\mathrm{CH}$ proton - 6.3-5.9 ppm, and methyl group of the pyran cycle $2.3-2.2 \mathrm{ppm}$ (Table). In addition, corresponding signals of methine protons and aryl substituents are also present in the spectra. The most sensitive to solvent changes is the $\mathrm{OH}$ proton bound by an intramolecular hydrogen bond to the carbonyl group of the pyran ring. Signals in DMSO are usually shifted by $0.1-1.0 \mathrm{ppm}$ in a stronger field compared to $\mathrm{CDCl}_{3}$ for dehydroacetic acid and chalcones based on it. $\mathrm{CH}$ proton signals are shifted by approximately $0.3 \mathrm{ppm}$ in a weaker field, and the signals of the protons of the methyl group are almost insensitive to the solvent.

The investigated compounds absorption maxima in DMF are located in the range of 348-490 nm (except for compounds with a nitro group, which have a maximum absorption in the UV region), with extinction coefficients $(\log \varepsilon)$ in the range of 3.93-4.46.

In methanol, the absorption maxima are located between 350 and $472 \mathrm{~nm}$, the extinction coefficients $(\log \varepsilon)$ are 3.66-4.54.

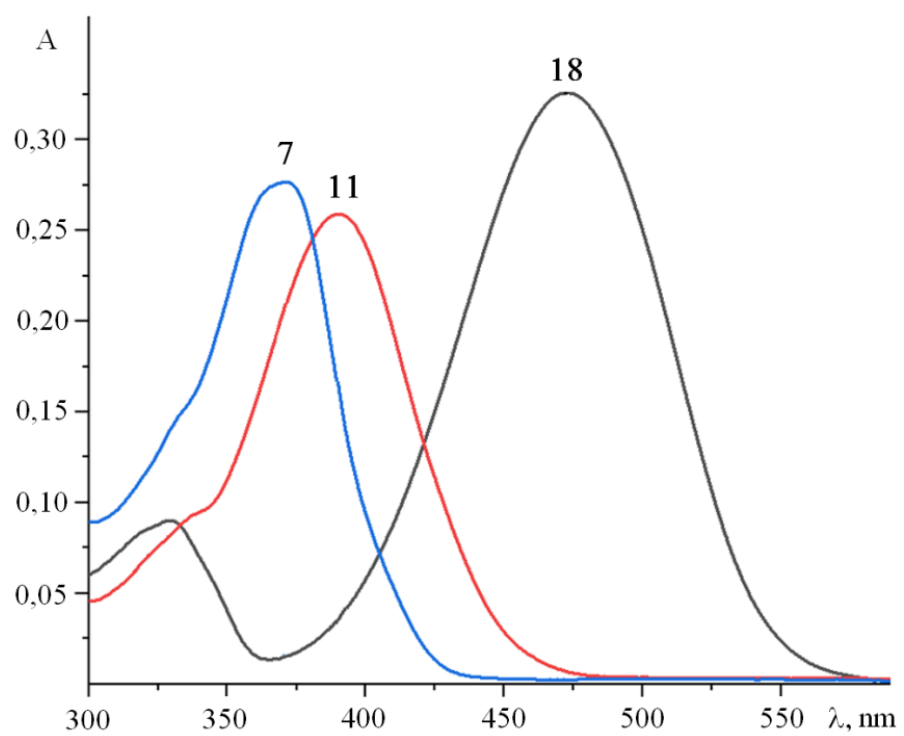

Fig. 4. UV-VIS spectra of chalcones 7,11 , and 18 in dimethylformamide $\left(\mathrm{C}=1 \bullet 10^{-4} \mathrm{M}\right)$.

In acetonitrile, the absorption maxima are in the range of $279-477 \mathrm{~nm}$, the extinction coefficients $(\log \varepsilon)$ are $3.55-4.75$. The electron-donor substituents in the phenyl ring $\left(-\mathrm{N}\left(\mathrm{CH}_{3}\right)_{2}\right.$ and $\left.-\mathrm{N}\left(\mathrm{C}_{2} \mathrm{H}_{5}\right)_{2}\right)$ shift the absorption maximum bathochromically by almost $100 \mathrm{~nm}$ compared to others in all investigated solvents.

Comparing the absorption spectra of one compound in different solvents lead to the conclusion that the solvatochromic effects for most of them are weak - the position of the maximum changes by less than $10 \mathrm{~nm}$. However, for compounds 10,13 , and 16 , the maximum is shifted to the red region in acetonitrile by $15-17 \mathrm{~nm}$ compared to DMF. Moreover, for compounds 12 and 15 the maximum is shifted to the red region by $19 \mathrm{~nm}$. The most significant shift is observed for the compound 20 by $30 \mathrm{~nm}$. Compound 21, in contrast, in DMF has a red-shifted maximum in comparison to acetonitrile, 381 and $345 \mathrm{~nm}$, respectively (table). 
Table

The proton signals of the pyran cycle in the ${ }^{1} \mathrm{H}$ NMR spectra and the UV-VIS data of the obtained chalcones.

\begin{tabular}{|c|c|c|c|c|c|c|c|c|c|}
\hline \multirow[t]{2}{*}{ № } & \multicolumn{6}{|c|}{${ }^{1} \mathrm{H}$ NMR, $\delta, \mathrm{ppm}$} & \multicolumn{3}{|c|}{ UV-VIS, $\lambda, n m(\log \varepsilon)$} \\
\hline & $\mathrm{CDCl}_{3}$ & $\begin{array}{l}\text { DMSO } \\
\mathrm{H}\end{array}$ & $\mathrm{CDCl}_{3}$ & $\begin{array}{l}\text { DMSO } \\
\mathrm{H}\end{array}$ & $\mathrm{CDCl}_{3}$ & $\begin{array}{l}\text { DMSO } \\
\mathrm{Me}\end{array}$ & DMF & $\mathrm{MeOH}$ & $\mathrm{MeCN}$ \\
\hline DHA & 16.67 & 16.54 & 5.92 & 6.28 & 2.26 & 2.25 & - & - & - \\
\hline 1 & 17.95 & 17.55 & 5.97 & 6.32 & 2.29 & 2.28 & $354(4,15)$ & $350(3,96)$ & $357(4,20)$ \\
\hline 2 & 17.44 & 16.94 & 6.01 & 6.36 & 2.31 & 2.28 & $357(4,03)$ & $354(3,66)$ & $356(3,87)$ \\
\hline 3 & 17.48 & 17.12 & 6.01 & 6.34 & 2.31 & 2.28 & $356(3,95)$ & $352(3,67)$ & $360(3,55)$ \\
\hline 4 & 17.42 & 17.04 & 6.01 & 6.34 & 2.32 & 2.28 & $326(4,22)$ & $360(3,49)$ & $361(3,55)$ \\
\hline 5 & - & 18.01 & - & 6.28 & - & 2.26 & $357(4,09)$ & $371(4,18)$ & $378(4,24)$ \\
\hline 6 & 17.86 & 17.66 & 5.95 & 6.28 & 2.27 & 2.26 & $474(3,96)$ & $358(4,22)$ & $361(4,09)$ \\
\hline 7 & 17.53 & 18.08 & 5.95 & 6.25 & 2.27 & 2.25 & $369(4,27)$ & $383(4,52)$ & $383(4,23)$ \\
\hline 8 & 18.13 & 17.78 & 5.93 & 6.29 & 2.26 & 2.26 & $367(4,17)$ & $371(4,12)$ & $376(4,21)$ \\
\hline 9 & 17.92 & 17.49 & 5.97 & 6.31 & 2.29 & 2.26 & $360(4,16)$ & $356(4,06)$ & $360(4,17)$ \\
\hline 10 & 18.18 & 17.89 & 5.94 & 6.27 & 2.27 & 2.25 & $366(4,21)$ & $375(4,51)$ & $381(4,75)$ \\
\hline 11 & 18.24 & 17.93 & 5.94 & 6.27 & 2.27 & 2.25 & $377(4,09)$ & $373(3,95)$ & $383(4,07)$ \\
\hline 12 & 18.16 & 17.80 & 5.96 & 6.18 & 2.26 & 2.18 & $374(4,22)$ & $391(4,26)$ & $393(4,49)$ \\
\hline 13 & 18.44 & 17.42 & 5.93 & 6.25 & 2.26 & 2.24 & $382(4,04)$ & $396(4,24)$ & $399(4,05)$ \\
\hline 14 & 17.80 & 17.32 & 5.90 & 6.29 & 2.22 & 2.29 & $360(4,16)$ & $357(4,10)$ & $360(4,14)$ \\
\hline 15 & 17.97 & 17.64 & 5.95 & 6.31 & 2.27 & 2.28 & $360(3,91)$ & $370(4,40)$ & $279(4,24)$ \\
\hline 16 & 18.11 & 17.78 & 5.94 & 6.28 & 2.27 & 2.25 & $372(4,16)$ & $381(4,15)$ & $387(3,96)$ \\
\hline 17 & 17.80 & 17.50 & 5.90 & 6.33 & 2.22 & 2.28 & $357(4,14)$ & $354(4,19)$ & $358(4,15)$ \\
\hline 18 & 18.61 & 18.57 & 5.89 & 6.22 & 2.24 & 2.25 & $\begin{array}{l}474(4,29) \\
347(4,14)\end{array}$ & $\begin{array}{l}456(4,45) \\
330(3,98)\end{array}$ & $465(4,25)$ \\
\hline 19 & 18.71 & 18.57 & 5.90 & 6.18 & 2.24 & 2.23 & $490(4,39)$ & $472(4,54)$ & $477(4,02)$ \\
\hline 20 & 17.98 & 17.51 & 5.98 & 6.34 & 2.29 & 2.28 & $348(4,08)$ & $378(4,22)$ & $378(3,88)$ \\
\hline 21 & 18.13 & 17.77 & 5.98 & 6.35 & 2.30 & 2.30 & $381(4,46)$ & $345(4,35)$ & $345(4,42)$ \\
\hline
\end{tabular}

The general synthesis method of dehydroacetic acid condensed derivatives. The reactions were performed by a slightly modified procedure given in the work [3]. $10 \mathrm{mmol}$ of substituted benzaldehyde was added to $10 \mathrm{mmol}$ of dehydroacetic acid in $10 \mathrm{ml}$ of $\mathrm{n}$-butanol and was heated up to boiling temperature. 10 drops of a mixture of pyridine and piperidine (1:1 by volume) were added to the boiling homogeneous solution and refluxed for 2-4 h. Half of the solvent was distilled, the solution was cooled down and filtered from fell-out crystals. The product was washed twice on the filter with a small amount of methanol and recrystallized from the DMF-ethanol system. After that, the product was filtered, washed on the filter with methanol, twice with hot water, and air-dried. Data from ${ }^{1} \mathrm{H}$ NMR and UV-VIS spectroscopy are given in the table. 
DHA (3-acetyl-4-hydroxy-6-methyl-2Hpyran-2-one). ${ }^{1} \mathrm{H}$ NMR (300 MHz, DMSO- $d_{6}$ ) $\delta 16.54(\mathrm{~s}, 1 \mathrm{H}), 6.28(\mathrm{~s}, 1 \mathrm{H}), 2.54(\mathrm{~s}, 3 \mathrm{H}), 2.25$ (s, 3H). ${ }^{1} \mathrm{H}$ NMR (300 MHz, $\left.\mathrm{CDCl}_{3}\right) \delta 16.67$ (s, 1H), 5.92 (s, 1H), 2.64 (s, 3H), 2.26 (s, 3H).

1) 3-cinnamoyl-4-hydroxy-6-methyl-2Hpyran- 2-one. Yield: $59 \%$. ${ }^{1} \mathrm{H}$ NMR $(400 \mathrm{MHz}$, DMSO- $\left.d_{6}\right) \delta 17.55(\mathrm{~s}, 1 \mathrm{H}), 8.17(\mathrm{~d}, J=15.8 \mathrm{~Hz}$, $1 \mathrm{H}), 7.91(\mathrm{~d}, J=15.9 \mathrm{~Hz}, 1 \mathrm{H}), 7.79-7.66(\mathrm{~m}$, $2 \mathrm{H}), 7.59-7.37(\mathrm{~m}, 3 \mathrm{H}), 6.32(\mathrm{~s}, 1 \mathrm{H}), 2.28$ (s, 3H). ${ }^{1} \mathrm{H}$ NMR (300 MHz, Chloroform-d) $\delta 17.95(\mathrm{~s}, 1 \mathrm{H}), 8.32(\mathrm{~d}, J=15.7 \mathrm{~Hz}, 1 \mathrm{H}), 7.97$ $(\mathrm{d}, J=15.7 \mathrm{~Hz}, 1 \mathrm{H}), 7.76-7.54(\mathrm{~m}, 2 \mathrm{H}), 7.51-$ $7.34(\mathrm{~m}, 3 \mathrm{H}), 5.97$ (s, 1H), $2.29(\mathrm{~s}, 3 \mathrm{H})$.

2) (E)-4-hydroxy-6-methyl-3-(3-(2-nitrophenyl)acryloyl)-2H-pyran-2-one. Yield: $43 \%$. ${ }^{1} \mathrm{H}$ NMR (500 MHz, DMSO- $\left.d_{6}\right) \delta 16.94$ (s, 1H), 8.20-7.99 (m, 3H), 7.95-7.81 (m, 2H), 7.73-7.62 (m, 1H), $6.36(\mathrm{~s}, 1 \mathrm{H}), 2.28$ (s, 3H). ${ }^{1} \mathrm{H}$ NMR (300 MHz, Chloroform-d) $\delta 17.44$ (s, $1 \mathrm{H}), 8.34(\mathrm{~d}, J=15.6 \mathrm{~Hz}, 1 \mathrm{H}), 8.22(\mathrm{~d}, J=$ $15.5 \mathrm{~Hz}, 1 \mathrm{H}), 8.05$ (d, $J=8.1 \mathrm{~Hz}, 1 \mathrm{H}), 7.85$ (d, $J=7.8 \mathrm{~Hz}, 1 \mathrm{H}), 7.76-7.64(\mathrm{~m}, 1 \mathrm{H}), 7.57(\mathrm{td}, J=$ 7.8, $1.5 \mathrm{~Hz}, 1 \mathrm{H}), 6.01$ (s, 1H), 2.31 (s, 3H).

3) (E)-4-hydroxy-6-methyl-3-(3-(3-nitrophenyl)acryloyl)-2H-pyran-2-one. Yield: $31 \%$. ${ }^{1} \mathrm{H}$ NMR (500 MHz, DMSO-d $) \delta 17.12$ (s, $1 \mathrm{H}), 8.52(\mathrm{~s}, 1 \mathrm{H}), 8.35-8.26(\mathrm{~m}, 1 \mathrm{H}), 8.25-8.08$ $(\mathrm{m}, 2 \mathrm{H}), 7.98(\mathrm{~d}, J=15.9 \mathrm{~Hz}, 1 \mathrm{H}), 7.78-7.68$ (m, 1H), 6.34 (s, 1H), 2.28 (s, 3H). ${ }^{1} \mathrm{H}$ NMR (300 MHz, Chloroform-d) $\delta 17.48$ (s, 1H), $8.47(\mathrm{t}, J=2.0 \mathrm{~Hz}, 1 \mathrm{H}), 8.39(\mathrm{~d}, J=15.8 \mathrm{~Hz}$, $1 \mathrm{H}), 8.26$ (dd, $J=8.2,3.3 \mathrm{~Hz}, 1 \mathrm{H}), 8.02(\mathrm{~d}, J=$ $7.8 \mathrm{~Hz}, 1 \mathrm{H}), 7.93$ (d, $J=15.8 \mathrm{~Hz}, 1 \mathrm{H}), 7.60$ (t, $J=8.0 \mathrm{~Hz}, 1 \mathrm{H}), 6.01(\mathrm{~s}, 1 \mathrm{H}), 2.31(\mathrm{~s}, 3 \mathrm{H})$.

4) (E)-4-hydroxy-6-methyl-3-(3-(4-nitrophenyl)acryloyl)-2H-pyran-2-one. Yield: $20 \%$. ${ }^{1} \mathrm{H}$ NMR (500 MHz, DMSO- $\left.d_{6}\right) \quad \delta 17.04$ (s, $1 \mathrm{H}), 8.29(\mathrm{~d}, J=8.3 \mathrm{~Hz}, 2 \mathrm{H}), 8.23(\mathrm{~d}, J=$ $15.8 \mathrm{~Hz}, 1 \mathrm{H}), 7.97$ (d, $J=8.2 \mathrm{~Hz}, 2 \mathrm{H}), 7.93$ (d,
$J=16.1 \mathrm{~Hz}, 1 \mathrm{H}), 6.34(\mathrm{~s}, 1 \mathrm{H}), 2.28(\mathrm{~s}, 3 \mathrm{H})$. ${ }^{1} \mathrm{H}$ NMR (300 MHz, Chloroform-d) $\delta 17.42$ $(\mathrm{s}, 1 \mathrm{H}), 8.41(\mathrm{~d}, J=15.8 \mathrm{~Hz}, 1 \mathrm{H}), 8.27(\mathrm{~d}, J=$ $8.7 \mathrm{~Hz}, 2 \mathrm{H}), 7.91$ (d, J=15.8 Hz, 1H), 7.82 (d, $J=8.7 \mathrm{~Hz}, 2 \mathrm{H}), 6.01(\mathrm{~s}, 1 \mathrm{H}), 2.32(\mathrm{~s}, 3 \mathrm{H})$.

5) E)-4-hydroxy-3-(3-(2-hydroxyphenyl) acryloyl)-6-methyl-2H-pyran-2-one. Yield:50\%. ${ }^{1} \mathrm{H}$ NMR (400 MHz, DMSO-d $) \delta 18.01(\mathrm{~s}, 1 \mathrm{H})$, $10.52(\mathrm{~s}, 1 \mathrm{H}), 8.53-7.86(\mathrm{~m}, 2 \mathrm{H}), 7.58(\mathrm{~d}, J=$ $7.9 \mathrm{~Hz}, 1 \mathrm{H}), 7.31(\mathrm{t}, J=15.7 \mathrm{~Hz}, 1 \mathrm{H}), 6.98-6.78$ $(\mathrm{m}, 2 \mathrm{H}), 6.28(\mathrm{~s}, 1 \mathrm{H}), 2.26(\mathrm{~s}, 3 \mathrm{H})$. Insoluble in chloroform-d.

6) (E)-4-hydroxy-3-(3-(3-hydroxyphenyl) acryloyl)-6-methyl-2H-pyran-2-one. Yield: $39 \%$. ${ }^{1} \mathrm{H}$ NMR (500 MHz, DMSO-d $) \delta 17.66$ (s, $1 \mathrm{H}), 9.77$ (s, 1H), $8.11(\mathrm{~d}, J=15.8 \mathrm{~Hz}, 1 \mathrm{H})$, $7.81(\mathrm{~d}, J=15.8 \mathrm{~Hz}, 1 \mathrm{H}), 7.28(\mathrm{t}, J=8.0 \mathrm{~Hz}$, $1 \mathrm{H}), 7.13(\mathrm{~d}, J=6.4 \mathrm{~Hz}, 2 \mathrm{H}), 6.89(\mathrm{dd}, 1 \mathrm{H})$, 6.28 (s, 1H), 2.26 (s, 3H). ${ }^{1} \mathrm{H}$ NMR (400 MHz, Chloroform- $d) \delta 17.86(\mathrm{~s}, 1 \mathrm{H}), 8.26(\mathrm{~d}, J=$ $15.7 \mathrm{~Hz}, 1 \mathrm{H}), 7.88$ (d, $J=15.7 \mathrm{~Hz}, 1 \mathrm{H}), 7.28$ $(\mathrm{t}, J=7.7 \mathrm{~Hz}, 3 \mathrm{H}), 7.15(\mathrm{~s}, 1 \mathrm{H}), 6.97-6.86(\mathrm{~m}$, $1 \mathrm{H}), 5.95$ (s, 1H), 2.27 (s, 2H).

7) (E)-4-hydroxy-3-(3-(4-hydroxyphenyl) acryloyl)-6-methyl-2H-pyran-2-one. Yield: $21 \% .{ }^{1} \mathrm{H}$ NMR (500 MHz, DMSO- $d_{6}$ ) $\delta 18.08$ (s, 1H), 10.35 (s, 1H), $8.01(\mathrm{~d}, J=15.7 \mathrm{~Hz}, 1 \mathrm{H})$, $7.88(\mathrm{~d}, J=15.7 \mathrm{~Hz}, 1 \mathrm{H}), 7.59(\mathrm{~d}, J=8.5 \mathrm{~Hz}$, $2 \mathrm{H}), 6.87(\mathrm{~d}, J=8.5 \mathrm{~Hz}, 2 \mathrm{H}), 6.25(\mathrm{~s}, 1 \mathrm{H}), 2.25$ (s, 3H). ${ }^{1} \mathrm{H}$ NMR (500 MHz, Chloroform-d) $\delta$ 17.53 (s, 1H), 8.20 (d, $J=16.0 \mathrm{~Hz}, 1 \mathrm{H}), 7.94$ (d, $J=15.7 \mathrm{~Hz}, 1 \mathrm{H}), 7.62(\mathrm{~d}, J=7.9 \mathrm{~Hz}, 2 \mathrm{H}), 6.88$ $(\mathrm{d}, J=8.0 \mathrm{~Hz}, 2 \mathrm{H}), 5.95$ (s, 1H), 2.27 (s, 3H).

8) (E)-4-hydroxy-3-(3-(2-methoxyphenyl) acryloyl)-6-methyl-2H-pyran-2-one. Yield: $68 \%$. ${ }^{1} \mathrm{H}$ NMR (400 MHz, DMSO-d $) \delta 17.78$ (s, 1H), $8.24(\mathrm{~d}, J=15.9 \mathrm{~Hz}, 1 \mathrm{H}), 8.15(\mathrm{~d}, J=16.0 \mathrm{~Hz}$, $1 \mathrm{H}), 7.67$ (dd, $J=7.7,1.7 \mathrm{~Hz}, 1 \mathrm{H}), 7.54-7.43$ $(\mathrm{m}, 1 \mathrm{H}), 7.13(\mathrm{~d}, J=8.4 \mathrm{~Hz}, 1 \mathrm{H}), 7.05(\mathrm{t}, J=$ $8.0 \mathrm{~Hz}, 1 \mathrm{H}), 6.29$ (s, 1H), 3.89 (s, 3H), 2.26 (s, 
3H). ${ }^{1} \mathrm{H}$ NMR (500 MHz, Chloroform-d) $\delta$ $18.13(\mathrm{~s}, 1 \mathrm{H}), 8.51-8.16(\mathrm{~m}, 2 \mathrm{H}), 7.72(\mathrm{~d}, J=$ $7.7 \mathrm{~Hz}, 1 \mathrm{H}), 7.38(\mathrm{t}, J=8.1 \mathrm{~Hz}, 1 \mathrm{H}), 6.98(\mathrm{t}, J=$ $7.5 \mathrm{~Hz}, 1 \mathrm{H}), 6.92(\mathrm{~d}, J=8.4 \mathrm{~Hz}, 1 \mathrm{H}), 5.93(\mathrm{~s}$, $1 \mathrm{H}), 3.91(\mathrm{~s}, 3 \mathrm{H}), 2.26(\mathrm{~s}, 3 \mathrm{H})$.

9) (E)-4-hydroxy-3-(3-(3-methoxyphenyl)acryloyl)-6-methyl-2H-pyran-2-one. Yield: $63 \% .{ }^{1} \mathrm{H}$ NMR (500 MHz, DMSO- $d_{6}$ ) $\delta 17.49$ (s, 1H), $8.13(\mathrm{~d}, J=16.1 \mathrm{~Hz}, 1 \mathrm{H}), 7.86(\mathrm{~d}, J=$ $15.8 \mathrm{~Hz}, 1 \mathrm{H}), 7.39$ (t, $J=7.9 \mathrm{~Hz}, 1 \mathrm{H}), 7.31$ (d, $J$ $=7.5 \mathrm{~Hz}, 1 \mathrm{H}), 7.24(\mathrm{~s}, 1 \mathrm{H}), 7.11-6.99(\mathrm{~m}, 1 \mathrm{H})$, $6.31(\mathrm{~s}, 1 \mathrm{H}), 3.79(\mathrm{~s}, 3 \mathrm{H}), 2.26$ (s, 3H). ${ }^{1} \mathrm{H}$ NMR (300 MHz, Chloroform-d) $\delta 17.92$ (s, 1H), 8.30 (d, $J=15.7 \mathrm{~Hz}, 1 \mathrm{H}), 7.93$ (d, $J=15.8 \mathrm{~Hz}, 1 \mathrm{H})$, $7.41-7.26(\mathrm{~m}, 2 \mathrm{H}), 7.23-7.13(\mathrm{~m}, 1 \mathrm{H}), 6.98$ (ddd, $J=7.7,2.6,1.5 \mathrm{~Hz}, 1 \mathrm{H}), 5.97$ (s, 1H), 3.85 (s, 3H), 2.29 (s, 3H).

10) (E)-4-hydroxy-3-(3-(4-methoxyphenyl)acryloyl)-6-methyl-2H-pyran-2-one. Yield: 49 \%. ${ }^{1} \mathrm{H}$ NMR (500 MHz, DMSO- $d_{6}$ ) $\delta 17.89(\mathrm{~s}, 1 \mathrm{H}), 8.05(\mathrm{~d}, J=15.7 \mathrm{~Hz}, 1 \mathrm{H}), 7.90$ $(\mathrm{d}, J=15.7 \mathrm{~Hz}, 1 \mathrm{H}), 7.69(\mathrm{~d}, J=8.4 \mathrm{~Hz}, 2 \mathrm{H})$, $7.04(\mathrm{~d}, J=8.2 \mathrm{~Hz}, 2 \mathrm{H}), 6.27$ (s, 1H), 3.81 (s, 3H), 2.25 (s, 3H). ${ }^{1} \mathrm{H}$ NMR (400 MHz, Chloroform- $d) \delta 18.18(\mathrm{~s}, 1 \mathrm{H}), 8.20(\mathrm{~d}, J=15.6$ $\mathrm{Hz}, 1 \mathrm{H}), 7.96$ (d, $J=15.7 \mathrm{~Hz}, 1 \mathrm{H}), 7.66$ (d, $J=$ $8.8 \mathrm{~Hz}, 2 \mathrm{H}), 6.93$ (d, $J=8.8 \mathrm{~Hz}, 2 \mathrm{H}), 5.94$ (s, $1 \mathrm{H}), 3.86$ (s, 3H), 2.27 (s, 3H).

11) (E)-4-hydroxy-6-methyl-3-(3-(4propoxyphenyl)acryloyl)-2H-pyran-2-one. Yield: $65 \%$. ${ }^{1} \mathrm{H}$ NMR (500 MHz, DMSO- $d_{6}$ ) $\delta 17.93(\mathrm{~s}, 1 \mathrm{H}), 8.04(\mathrm{~d}, J=15.8 \mathrm{~Hz}, 1 \mathrm{H}), 7.90$ $(\mathrm{d}, J=15.7 \mathrm{~Hz}, 1 \mathrm{H}), 7.67(\mathrm{~d}, J=8.2 \mathrm{~Hz}, 2 \mathrm{H})$, $7.02(\mathrm{~d}, J=8.3 \mathrm{~Hz}, 2 \mathrm{H}), 6.27(\mathrm{~s}, 1 \mathrm{H}), 3.98(\mathrm{t}, J$ $=6.4 \mathrm{~Hz}, 2 \mathrm{H}), 2.25(\mathrm{q}, 3 \mathrm{H}), 1.73(\mathrm{q}, J=7.1 \mathrm{~Hz}$, $2 \mathrm{H}), 0.96(\mathrm{t}, J=7.2 \mathrm{~Hz}, 3 \mathrm{H}) .{ }^{1} \mathrm{H} \operatorname{NMR}(300$ $\mathrm{MHz}$, Chloroform-d) $\delta 18.24(\mathrm{~s}, 1 \mathrm{H}), 8.20$ (d, $J=15.6 \mathrm{~Hz}, 1 \mathrm{H}), 7.96(\mathrm{~d}, J=15.6 \mathrm{~Hz}, 1 \mathrm{H})$, 7.70-7.60 (m, 2H), 6.97-6.88 (m, 2H), $5.94(\mathrm{~s}$, $1 \mathrm{H}), 3.97(\mathrm{t}, J=6.6 \mathrm{~Hz}, 2 \mathrm{H}), 2.27(\mathrm{~s}, 3 \mathrm{H}), 1.83$ $(\mathrm{q}, J=7.1,6.7 \mathrm{~Hz}, 2 \mathrm{H}), 1.05(\mathrm{t}, J=7.4 \mathrm{~Hz}, 3 \mathrm{H})$.

12) (E)-4-hydroxy-3-(3-(4-hydroxy-3methoxyphenyl)acryloyl)-6-methyl-2Hpyran-2-one. Yield: 43 \%. ${ }^{1} \mathrm{H}$ NMR $(500 \mathrm{MHz}$, DMSO-d $d_{6} \delta 17.80(s, 1 \mathrm{H}), 9.87(\mathrm{~s}, 1 \mathrm{H}), 7.93$ $(\mathrm{d}, J=15.6 \mathrm{~Hz}, 1 \mathrm{H}), 7.81(\mathrm{~d}, J=15.6 \mathrm{~Hz}, 1 \mathrm{H})$, $7.42-7.05(\mathrm{~m}, 2 \mathrm{H}), 6.80(\mathrm{~d}, J=8.1 \mathrm{~Hz}, 1 \mathrm{H})$, 6.18 (s, 1H), 3.75 (s, 3H), 2.18 (s, 3H). ${ }^{1} \mathrm{H}$ NMR $(500 \mathrm{MHz}$, Chloroform-d) $\delta 18.16(\mathrm{~s}, 1 \mathrm{H}), 8.16$ $(\mathrm{d}, J=15.6 \mathrm{~Hz}, 1 \mathrm{H}), 7.92(\mathrm{~d}, J=15.6 \mathrm{~Hz}, 1 \mathrm{H})$, 7.24-7.14 (m, 2H), $6.94(\mathrm{~d}, J=8.2 \mathrm{~Hz}, 1 \mathrm{H})$, 5.96 (s, 1H), 5.94 (s, 1H), 3.96 (s, 3H), 2.26 (s, $3 \mathrm{H})$.

13) (E)-3-(3-(2,4-dimethoxyphenyl)acryloyl)-4-hydroxy-6-methyl-2H-pyran-2-one. Yield: $51 \%$. ${ }^{1} \mathrm{H}$ NMR (500 MHz, DMSO- $d_{6}$ ) $\delta 17.42(\mathrm{~s}, 1 \mathrm{H}), 8.13(\mathrm{~s}, 2 \mathrm{H}), 7.76-7.49(\mathrm{~m}, 1 \mathrm{H})$, 6.79-6.51 (m, 2H), 6.25 (s, 1H), 3.89 (s, 3H), 3.83 (s, 3H), 2.24 (s, 3H). ${ }^{1} \mathrm{H}$ NMR $(300 \mathrm{MHz}$, Chloroform- $d$ ) $\delta 18.44(\mathrm{~s}, 1 \mathrm{H}), 8.31(\mathrm{~d}, J=$ $4.0 \mathrm{~Hz}, 2 \mathrm{H}), 7.69$ (d, J = 8.8 Hz, 1H), 6.55 (dd, $J=8.6,2.4 \mathrm{~Hz}, 1 \mathrm{H}), 6.45(\mathrm{~d}, J=2.4 \mathrm{~Hz}, 1 \mathrm{H}), 5.93$ (s, 1H), $3.91(\mathrm{~s}, 3 \mathrm{H}), 3.87(\mathrm{~s}, 3 \mathrm{H}), 2.26(\mathrm{~s}, 3 \mathrm{H})$.

14) (E)-3-(3-(3,5-dimethoxyphenyl)acryloyl)-4-hydroxy-6-methyl-2H-pyran-2-one. Yield: $52 \% .{ }^{1} \mathrm{H}$ NMR (400 MHz, DMSO- $d_{6}$ ) $\delta 17.32(\mathrm{~s}, 1 \mathrm{H}), 8.09(\mathrm{~d}, J=15.8 \mathrm{~Hz}, 1 \mathrm{H}), 7.82$ $(\mathrm{d}, J=15.8 \mathrm{~Hz}, 1 \mathrm{H}), 6.87(\mathrm{~s}, 2 \mathrm{H}), 6.64(\mathrm{~s}, 1 \mathrm{H})$, $6.29(\mathrm{~s}, 1 \mathrm{H}), 3.81(\mathrm{~s}, 6 \mathrm{H}), 2.29$ (s, 3H). ${ }^{1} \mathrm{H}$ NMR (500 MHz, Chloroform-d) $\delta 17.80(\mathrm{~s}, 1 \mathrm{H}), 8.20$ (d, $J=15.7 \mathrm{~Hz}, 1 \mathrm{H}), 7.81$ (d, $J=15.7 \mathrm{~Hz}, 1 \mathrm{H})$, 6.75 (s, 2H), 6.46 (s, 1H), 5.90 (s, 1H), 3.77 (s, $6 \mathrm{H}), 2.22(\mathrm{~s}, 3 \mathrm{H})$.

15) (E)-4-hydroxy-6-methyl-3-(3-(3,4,5trimethoxyphenyl)acryloyl)-2H-pyran-2-one. Yield: $51 \%$. ${ }^{1} \mathrm{H}$ NMR (400 MHz, DMSO- $d_{6}$ ) $\delta 17.64(\mathrm{~s}, 1 \mathrm{H}), 8.05(\mathrm{~d}, J=15.5 \mathrm{~Hz}, 1 \mathrm{H}), 7.87$ $(\mathrm{d}, J=15.7 \mathrm{~Hz}, 1 \mathrm{H}), 7.06(\mathrm{~s}, 2 \mathrm{H}), 6.31(\mathrm{~s}, 1 \mathrm{H})$, $3.84(\mathrm{~s}, 6 \mathrm{H}), 3.73$ (s, 3H), 2.28 (s, 3H). ${ }^{1} \mathrm{H}$ NMR $(500 \mathrm{MHz}$, Chloroform-d) $\delta 17.97$ (s, 1H), 8.20 
$(\mathrm{d}, J=15.6 \mathrm{~Hz}, 1 \mathrm{H}), 7.88(\mathrm{~d}, J=15.7 \mathrm{~Hz}, 1 \mathrm{H})$, $6.89(\mathrm{~s}, 2 \mathrm{H}), 5.95(\mathrm{~s}, 1 \mathrm{H}), 3.91(\mathrm{~s}, 6 \mathrm{H}), 3.89$ (s, $3 \mathrm{H}), 2.27(\mathrm{~s}, 3 \mathrm{H})$.

16) (E)-3-(3-(benzo[d][1,3]dioxol-5-yl) acryloyl)-4-hydroxy-6-methyl-2H-pyran2-one. Yield: $75 \%$. ${ }^{1} \mathrm{H}$ NMR $(500 \mathrm{MHz}$, DMSO- $\left.d_{6}\right) \delta 17.78(\mathrm{~s}, 1 \mathrm{H}), 7.99(\mathrm{~d}, J=15.7$ $\mathrm{Hz}, 1 \mathrm{H}), 7.85$ (d, $J=15.7 \mathrm{~Hz}, 1 \mathrm{H}), 7.43-7.14$ $(\mathrm{m}, 2 \mathrm{H}), 7.01(\mathrm{~d}, J=8.0 \mathrm{~Hz}, 1 \mathrm{H}), 6.28(\mathrm{~s}, 1 \mathrm{H})$, $6.11(\mathrm{~s}, 2 \mathrm{H}), 2.25(\mathrm{~s}, 3 \mathrm{H}) .{ }^{1} \mathrm{H}$ NMR $(300 \mathrm{MHz}$, Chloroform- $d) \delta 18.11(\mathrm{~s}, 1 \mathrm{H}), 8.14(\mathrm{~d}, J=$ $15.6 \mathrm{~Hz}, 1 \mathrm{H}), 7.88(\mathrm{~d}, J=15.6 \mathrm{~Hz}, 1 \mathrm{H}), 7.22(\mathrm{~d}$, $J=1.8 \mathrm{~Hz}, 1 \mathrm{H}), 7.16(\mathrm{dd}, J=8.1,1.7 \mathrm{~Hz}, 1 \mathrm{H})$, $6.83(\mathrm{~d}, J=8.0 \mathrm{~Hz}, 1 \mathrm{H}), 6.03(\mathrm{~s}, 2 \mathrm{H}), 5.94(\mathrm{~s}$, $1 \mathrm{H}), 2.27(\mathrm{~s}, 3 \mathrm{H})$.

17) (E)-3-(3-(4-fluorophenyl)acryloyl)4-hydroxy-6-methyl-2H-pyran-2-one. Yield: 49 \%. ${ }^{1} \mathrm{H}$ NMR (300 MHz, DMSO- $d_{6}$ ) $\delta 17.50$ $(\mathrm{s}, 1 \mathrm{H}), 8.12(\mathrm{~d}, J=15.8 \mathrm{~Hz}, 1 \mathrm{H}), 7.92(\mathrm{~d}, J=$ $15.9 \mathrm{~Hz}, 1 \mathrm{H}), 7.88-7.74(\mathrm{~m}, 2 \mathrm{H}), 7.34(\mathrm{t}, J=$ $8.8 \mathrm{~Hz}, 2 \mathrm{H}), 6.33(\mathrm{~s}, 1 \mathrm{H}), 2.28(\mathrm{~s}, 3 \mathrm{H}) .{ }^{1} \mathrm{H}$ NMR $(500 \mathrm{MHz}$, Chloroform- $d$ ) $\delta 17.80(\mathrm{~s}, 1 \mathrm{H}), 8.17$ $(\mathrm{d}, J=15.7 \mathrm{~Hz}, 1 \mathrm{H}), 7.85(\mathrm{~d}, J=15.7 \mathrm{~Hz}, 1 \mathrm{H})$, $7.62(\mathrm{dd}, J=8.5,5.5 \mathrm{~Hz}, 2 \mathrm{H}), 7.04(\mathrm{t}, J=8.4 \mathrm{~Hz}$, $2 \mathrm{H}), 5.90(\mathrm{~s}, 1 \mathrm{H}), 2.22(\mathrm{~s}, 3 \mathrm{H})$.

18) (E)-3-(3-(4-(dimethylamino)phenyl) acryloyl)-4-hydroxy-6-methyl-2H-pyran2-one. Yield: $32 \%$. ${ }^{1} \mathrm{H}$ NMR $(500 \mathrm{MHz}$, DMSO- $\left.d_{6}\right) \delta 18.57(\mathrm{~s}, 1 \mathrm{H}), 7.95(\mathrm{~s}, 2 \mathrm{H}), 7.59$ $(\mathrm{d}, J=9.0 \mathrm{~Hz}, 2 \mathrm{H}), 6.79(\mathrm{~d}, J=9.0 \mathrm{~Hz}, 2 \mathrm{H})$, $6.22(\mathrm{~s}, 1 \mathrm{H}), 3.34(\mathrm{~s}, 6 \mathrm{H}), 2.25$ (s, 3H). ${ }^{1} \mathrm{H}$ NMR $(500 \mathrm{MHz}$, Chloroform- $d) \delta 18.61(\mathrm{~s}, 1 \mathrm{H}), 8.10$ $(\mathrm{d}, J=15.4 \mathrm{~Hz}, 1 \mathrm{H}), 7.99(\mathrm{~d}, J=15.3 \mathrm{~Hz}, 1 \mathrm{H})$, $7.60(\mathrm{~d}, J=8.9 \mathrm{~Hz}, 2 \mathrm{H}), 6.67(\mathrm{~d}, J=9.0 \mathrm{~Hz}, 2 \mathrm{H})$, $5.89(\mathrm{~s}, 1 \mathrm{H}), 3.06(\mathrm{~s}, 6 \mathrm{H}), 2.24(\mathrm{~s}, 3 \mathrm{H})$.

19) (E)-3-(3-(4-(diethylamino)phenyl)acryloyl)-4-hydroxy-6-methyl-2H-pyran-2-one. Yield: $49 \% .{ }^{1} \mathrm{H}$ NMR $\left(500 \mathrm{MHz}, \mathrm{DMSO}-d_{6}\right)$ $\delta 18.57(\mathrm{~s}, 1 \mathrm{H}), 7.92(\mathrm{~s}, 2 \mathrm{H}), 7.54(\mathrm{~d}, J=8.6$ $\mathrm{Hz}, 2 \mathrm{H}), 6.74(\mathrm{~d}, J=8.5 \mathrm{~Hz}, 2 \mathrm{H}), 6.18(\mathrm{~s}, 1 \mathrm{H})$, $3.42(\mathrm{q}, J=7.0 \mathrm{~Hz}, 4 \mathrm{H}), 2.23(\mathrm{~s}, 3 \mathrm{H}), 1.11(\mathrm{t}$, $J=6.9 \mathrm{~Hz}, 6 \mathrm{H}) .{ }^{1} \mathrm{H}$ NMR $(500 \mathrm{MHz}$, Chloroform-d) $\delta 18.71(\mathrm{~s}, 1 \mathrm{H}), 8.36-7.79(\mathrm{~m}, 2 \mathrm{H})$, $7.59(\mathrm{~d}, J=9.0 \mathrm{~Hz}, 2 \mathrm{H}), 6.65(\mathrm{~d}, J=8.5 \mathrm{~Hz}, 2 \mathrm{H})$, $5.90(\mathrm{~d}, J=0.9 \mathrm{~Hz}, 1 \mathrm{H}), 3.43(\mathrm{q}, J=7.1 \mathrm{~Hz}, 4 \mathrm{H})$, $2.24(\mathrm{~s}, 3 \mathrm{H}), 1.21(\mathrm{t}, J=7.1 \mathrm{~Hz}, 6 \mathrm{H})$

20) (E)-4-hydroxy-6-methyl-3-(3-(naphthalen-1-yl)acryloyl)-2H-pyran-2-one. Yield: $31 \% .{ }^{1} \mathrm{H}$ NMR (500 MHz, DMSO- $\left.d_{6}\right) \delta 17.51$ $(\mathrm{s}, 1 \mathrm{H}), 8.67(\mathrm{~d}, J=15.6 \mathrm{~Hz}, 1 \mathrm{H}), 8.39-8.16(\mathrm{~m}$, $2 \mathrm{H}), 8.08(\mathrm{~d}, J=8.1 \mathrm{~Hz}, 1 \mathrm{H}), 7.99(\mathrm{dd}, J=22.5$, $7.1 \mathrm{~Hz}, 2 \mathrm{H}), 7.72-7.50(\mathrm{~m}, 3 \mathrm{H}), 6.34(\mathrm{~s}, 1 \mathrm{H}), 2.28$ (s, 3H). ${ }^{1} \mathrm{H}$ NMR (300 MHz, Chloroform- $d$ ) $\delta$ $17.98(\mathrm{~s}, 1 \mathrm{H}), 8.85(\mathrm{~d}, J=15.5 \mathrm{~Hz}, 1 \mathrm{H}), 8.42(\mathrm{~d}, J$ $=15.5 \mathrm{~Hz}, 1 \mathrm{H}), 8.29(\mathrm{~d}, J=7.7 \mathrm{~Hz}, 1 \mathrm{H}), 8.04(\mathrm{~d}, J$ = 7.3 Hz, 1H), 7.99-7.80 (m, 2H), 7.66-7.50 (m, $3 \mathrm{H}), 5.98(\mathrm{~s}, 1 \mathrm{H}), 2.29(\mathrm{~s}, 3 \mathrm{H})$.

21) (E)-4-hydroxy-6-methyl-3-(3-(pyren1-yl)acryloyl)-2H-pyran-2-one. Yield: $68 \%$. ${ }^{1} \mathrm{H}$ NMR (300 MHz, DMSO- $\left.d_{6}\right) \delta 17.77(\mathrm{~s}, 1 \mathrm{H})$, $9.02(\mathrm{~d}, J=15.4 \mathrm{~Hz}, 1 \mathrm{H}), 8.64(\mathrm{~d}, J=9.4 \mathrm{~Hz}$, $1 \mathrm{H}), 8.53-8.36(\mathrm{~m}, 6 \mathrm{H}), 8.35-8.20(\mathrm{~m}, 3 \mathrm{H})$, $6.35(\mathrm{~s}, 1 \mathrm{H}), 2.30(\mathrm{~s}, 3 \mathrm{H}) .{ }^{1} \mathrm{H}$ NMR $(500 \mathrm{MHz}$, Chloroform- $d$ ) $\delta 18.13(\mathrm{~s}, 1 \mathrm{H}), 9.17(\mathrm{~d}, J=15.4$ $\mathrm{Hz}, 1 \mathrm{H}), 8.68-8.49(\mathrm{~m}, 3 \mathrm{H}), 8.30-7.99(\mathrm{~m}$, $7 \mathrm{H}), 5.98(\mathrm{~s}, 1 \mathrm{H}), 2.30(\mathrm{~s}, 3 \mathrm{H})$.

\section{CONCLUSIONS}

The optimal conditions for the Knoevenagel reaction based on dehydroacetic acid and various aldehydes were determined. Twenty-one chalcones with substituents of different nature in the aromatic nucleus were obtained and characterized by ${ }^{1} \mathrm{H}$ NMR spectroscopy. The optical properties of synthesized compounds were investigated in $\mathrm{DMF}, \mathrm{MeOH}, \mathrm{MeCN}$. It was found that the absorption maxima are in the visible range from 330 to $490 \mathrm{~nm}$ with a weak solvatochromic effect for these compounds in studied solvents. 
The work was supported by the NAS of Ukraine project № 07-03-20/21 of joint competition of projects of the NAS of Ukraine - NAS of Belarus.

\section{СИНТЕЗ ТА ВЛАСТИВОСТІ ХАЛКОНІВ}

\section{НА ОСНОВІ ДЕГІДРАЦЕТОВОї КИСЛОТИ}

В. Я. Черній ${ }^{1^{*}}$, I. М. Третякова ${ }^{1}$, Н. М. Федосова ${ }^{1}$, I. М. Денисенко ${ }^{1}$, Я. М. Довбій ${ }^{1}$ В. Б. Ковальська ${ }^{1,2}$, С. В. Черній ${ }^{1,2}$, В. I. Пехньо ${ }^{1}$, А. С. Старухін ${ }^{3}$

${ }^{1}$ Інститут загальної та неорганічної хімії імені В. І. Вернадського НАН Украйни, Київ, Україна

${ }^{2}$ Інститут молекулярної біології та генетики НАН України, Київ, Україна

${ }^{3}$ Інститут фізики ім. Б. І. Степанова НАН Білорусі, Мінськ, Білорусь

*e-mail:v.chernii@gmail.com

У роботі досліджено реакцію конденсації Кневенагеля між дегідрацетовою кислотою та ароматичними альдегідами. Отримано 21 халкон із замісниками різної природи. Встановлено склад та будову отриманих сполук, досліджено їні оптичні властивості в різних розчинниках. Синтезовані халкони поглинають світло у видимому діапазоні 330-490 нм із молярними коефіцієнтами екстинкції 3,5-4,5.

Ключові слова: дегідрацетова кислота, конденсація Кневенагеля, халкони.

\section{REFERENCES}

1. Hale William J. The constitution of dehydroacetic acid. J. Am. Chem. Soc. 1911. 33(7): 1119-1135. https://doi.org/10.1021/ ja02220a015

2. Bhat A.N., Jain B.D. Separation and determination of uranium and thorium with 3-acetyl-4-hydroxy-coumarin. Talanta. 1960. 4: 13-16. https://doi.org/10.1016/ 0039-9140(60)80066-5

3. Dovbii Ya.M., Chernii V.Ya., Tretyakova I.M., Gorski A.V., Starukhin A.S., Volkov S.V. Synthesis of dehydroacetic acid derivatives with chromophoric chain and their complexes with zirconium phthalocyanine. Ukr. Chem. Journ. 2015. 81(12): 79-82.

4. Bhat A.N., Jain B.D. Gravimetric determination of cerium(IV) and its separation from rare earths using 3-acetyl-4-hydroxycoumarin. J. Less Common. Metals. 1961. 3: 259-261.

5. Bhat A.N., Jain B.D. Spectrophotometric studies of uranium (VI)-3-acetyl-4-hydroxycoumarin complex in ethanol. J. Inorg. Nucl. Chem. 1961. 23: 136-139. https:// doi.org/10.1016/0022-1902(61)80095-X

6. Hsieh W.Y., Zaleski C.M.. Pecoraro V.L., Liu S. Mn(II) Complexes of Monoanionic BidentateChelators: Thex-rayCrystal Structures of $\mathrm{Mn}(\mathrm{DHA})_{2}\left(\mathrm{CH}_{3} \mathrm{OH}\right)_{2} \quad(\mathrm{DHA}=-$ Dehydroacetic Acid) and $\left[\mathrm{Mn}(\mathrm{ema})_{2}\right.$ $\left.\left(\mathrm{H}_{2} \mathrm{O}\right)\right]_{2} \quad 2 \mathrm{H}_{2} \mathrm{O} \quad(\mathrm{Hema}=$ 2-Ethyl-3-Hydroxy-4-Pyrone). Inorg. Chim. Acta. 2006. 359: 228-236. https://doi.org/10.1016/j.ica. 2005.09.025

7. Chalaca M.Z., Figueroa-Villar J.D., Ellena J.A., Castellano E.E. Synthesis and structure of cadmium and zinc complexes 
of dehydroacetic acid. Inorg. Chim. Acta. 2002. 328: 45-52. https://doi.org/10.1016/ S0020-1693(01)00672-7

8. Jilalat A., Al-Garadi W., Karrouchi K., Essassi E. Dehydroacetic acid (part 1): chemical and pharmacological properties. J. Mar. Chim. Heterocycl. 2017. 16: 1-47. https://doi.org/10.48369/IMIST.PRSM/ jmch-v16il.8199

9. Walker G. N. Reduction of Enols. New Synthesis of Certain Methoxybenzsuberenes via Hydrogenation of Dehydroacetic Acids. Journal of the American Chemical Society. 1956. 78: 3201-3205. https://doi. org/10.1021/ja01594a062

10. Rehse K., Rüther D. Einfluß der S-Oxidation auf anticoagulante Wirkungen bei 4-Hydroxycumarinen, 4-Hydroxy-2-pyronen und 1,3-Indandionen. Archiv der Pharmazie, 1984. 317: 262-267. https:// doi.org/10.1002/ardp.19843170313

11. Yi Y. Y., He J. J., Su J. Q., Kong S. Z., Su J. Y., Li Y. C., Huang S. H., Li C. W., Lai X. P., Su Z. R. Synthesis and antimicrobial evaluation of pogostone and its analogues. Fitoterapia, 2013. 84: 135-139. https://doi.org/10.1016/j.fitote.2012.11.005

12. Aï-Baziz N., Rachedi Y., Chemat F., Hamdi M. Solvent Free Microwave-Assisted Knoevenagel Condensation of Dehydroacetic Acid with Benzaldehyde Derivatives. Asian Journal of Chemistry. 2008. 20: 2610-2622.

13. Rachedi Y., Hamdi M., Spéziale V. Synthesis of 4-Hydroxy 6-Methyl 3- $\beta$-Arylpropionyl 2-Pyrones by Selective Catalytic Hydrogenation of 3-Cinnamoyl 4-Hydroxy 6-Methyl 2-Pyrones. Synthetic Commun. 1989. 19: 3437-3442. https://doi. org/10.1080/00397918908052752
14. Sundar P. S., Gunasheela D. Synthesis, characterization and determination of antimicrobial activity of novel chalcones of 3-acetyl 4-hydroxy-6-methyl-2hpyran-2one. Europ. J. Pharm. Med. Research. 2016. 3(7): 381-384.

15. Patange V., Arbad B. Synthesis, spectral, thermal and biological studies of transition metal complexes of 4-hydroxy-3-[3-(4-hydroxyphenyl)-acryloyl]-6-methyl-pyran2-one. J. Serb. Chem. Soc. 2011. 76: 12371246. doi: 10.2298/JSC100531108P

16. Tambov K. V., Voevodina I. V., Manaev A. V., Ivanenkov Y. A.. Neamati N., Traven V. F. Structures and biological activity of cinnamoyl derivatives of coumarins and dehydroacetic acid and their boron difluoride complexes. Russ. Chem. Bul. 2012. 61: 78-90. https://doi.org/10.1007/s11172012-0012-y

17. Hanuza J., Ptak M., Lisiecki R., Janczak J., Kwocz A., Kucharska E., Roszak S., Ryba-Romanowski W., Mączka M., Hermanowicz K., Macalik L. Spectral and energetic transformation of femtosecond light impulses in the $\mathrm{Eu}^{3+}$ complex with dehydroacetic acid. J. Lumines. 2018. 198: 471-481. https://doi.org/10.1016/j.jlumin. 2018.02.067

18. Wu B., Wang Y., Chen S., Wang M., Ma M., Shi Y., Wang X. Stability, mechanism and unique "zinc burning" inhibition synergistic effect of zinc dehydroacetate as thermal stabilizer for poly(vinyl chloride). Polymer Degradation and Stability. 2018. 152: 228-234. DOI: 10.1016/j.polymdegradstab.2018.04.025

19. Malik B.A., Mir J.M. Synthesis, characterization and DFT aspects of some oxovanadium(IV) and manganese(II) complexes 
involving dehydroacetic acid and $\beta$-diketones. J.Coord. Chem. 2018. 71: 104-119. https://doi.org/10.1080/00958972.2018.14 29600

20. Vashisht D., Sharma S., Kumar R., Saini V., Saini V., Ibhadon A., Sahoo S.C., Sharma S., Mehta, S.K., Kataria, R. Dehydroacetic acid derived Schiff base as selective and sensitive colorimetric chemosensor for the detection of $\mathrm{Cu}(\mathrm{II})$ ions in aqueous medium. Microchemical Journal. 2020. 155: 1-10. https:// doi.org/10.1016/j.microc.2020.104705

21. Chen K.-H., Lin T.-H., Hsu T.-E., Li Y.-J., Chen G.-H., Leu W.-J., Guh J.-H., Lin C.-H., Huang J.-H. Ruthenium (II) complexes containing dehydroacetic acid and its imine derivative ligands. Synthesis, characterization and cancer cell growth anti-proliferation activity (GI50) study. J. Organometall. Chem. 2018. 871: 150-158. https://doi. org/10.1016/j.jorganchem.2018.07.014

22. Kendur U., Chimmalagi G.H., Patil S.M., Gudasi K.B., Frampton C.S. Synthesis, structural characterization and biological evaluation of mononuclear transition metal complexes of zwitterionic dehydroacetic acid N-aroylhydrazone ligand. Appl. Organometall. Chem. 2018. 32: 1-21. https://doi. org/10.1002/aoc.4278

23. Emam S.M., El-Tabl A.S., Ahmed H.M., Emad E.A. Synthesis, structural characterization, electrochemical and biological studies on divalent metal chelates of a new ligand derived from pharmaceutical preservative, dehydroacetic acid, with 1,4-diaminobenzene. Arabian J. Chem. 2017. 10: S3816 - S3825. DOI: 10.1016/j.arabjc.2014.05.019

24. Devi J., Devi S., Kumar A. Synthesis, spectral, and in vitro antimicrobial studies of
organosilicon(IV) complexes with Schiff bases derived from dehydroacetic acid. Monatshefte fur Chemie. 2016. 147: 21952207. https://doi.org/10.1007/s00706-0161720-z

25. Benferrah N., Hammadi M., Philouze C., Berthiol F., Thomas F. Copper(II) complex of a Schiff base of dehydroacetic acid: Characterization and aerobic oxidation of benzyl alcohol. Inorg. Chem. Commun. 2016. 72: 17-22. https://doi.org/10.1016/j. inoche.2016.07.020

26. Mir J.M., Rajak D.K., Maurya R.C. Oxovanadium(IV) complex of 8-hydroxy quinoline and 3-acetyl-6-methyl-2H-pyran2,4(3H)-dione: Experimental, theoretical and antibacterial evaluation. Journal of King Saud University - Science. 2019. 31: 1034-1041. https://doi.org/10.1016/j.jksus.2018.03.023

27. Sarhan A.M., Elsayed S.A., Mashaly M.M., El-Hendawy A.M. Oxovanadium(IV) and ruthenium(II) carbonyl complexes of ONS-donor ligands derived from dehydroacetic acid and dithiocarbazate: Synthesis, characterization, antioxidant activity, DNA binding and in vitro cytotoxicity. Appl. Organometall. Chem. 2019. 33: 1-16. № e4655. https://doi.org/10.1002/aoc.4655

28. Saini S., Pal R., Gupta A.K., Beniwal V. Synthesis, characterization, DNA photocleavage and antibacterial study of a novel dehydroacetic acid based hydrazone Schiff's base transition metal complexes. Res. J. Chem. Environ. 2017. 21: 49-57.

29. Nechak R., Bouzroura S. A., Benmalek Y., Salhi L., Martini S. P., Morizur V., Dunach, E., Kolli, B. N. Synthesis and Antimicrobial Activity Evaluation of Novel 4-Thiazolidinones Containing a Pyrone Moiety. 
Synthetic Commun. 2015. 45: 262-272. https://doi.org/10.1080/00397911.2014.97 0278

30. Swamy P. G., Sri B. R., Giles D., Shashidhar B., Das A. K., Agasimundin Y. Synthesis, anticancer, and molecular docking studies of pyranone derivatives. Med. Chem. Research. 2013. 22: 4909-4919. https://doi. org/10.1007/s00044-013-0478-7

31. Kashar T. I., El-Sehli A. H. Synthesis, characterization, antimicrobial and anticancer activity of $\mathrm{Zn}$ (II), $\mathrm{Pd}(\mathrm{II})$ and $\mathrm{Ru}(\mathrm{III})$ complexes of dehydroacetic acid hydrazine. Journal of Chemical and Pharmaceutical Research. 2013. 5(11): 474-483.

32. Thaisrivongs S., Romero D. L., Tommasi R. A., Janakiraman M. N., Strohbach J. W., Turner S. R., Biles C., Morge R. R., Johnson P. D., Aristoff P. A., Tomich P. K., Lynn J. C., Horng M. M., Chong K. T., Hinshaw R. R., Howe W. J., Finzel B. C., Watenpaugh K. D. Structure-Based Design of HIV Protease Inhibitors: 5,6-Dihydro-4-hydroxy-2-pyrones as Effective, Nonpeptidic Inhibitors. J. Med. Chem. 1996. 39: 46304642. doi: $10.1021 / \mathrm{jm} 960228 \mathrm{q}$.

33. Defant A., Mancini I., Tomazzolli R., Balzarini J. Design, Synthesis, and Biological
Evaluation of Novel 2H-Pyran-2-one Derivatives as Potential HIV-1 Reverse Transcriptase Inhibitors. Archiv Der Pharmazie. 2014. 348: 23-33. https://doi.org/10.1002/ ardp. 201400235

34. Kovalska V., Chernii S., Losytskyy M., Dovbii Y., Tretyakova I., Czerwieniec R., Chernii V., Yarmoluk S., Volkov S. $\beta$-ketoenole dyes: Synthesis and study as fluorescent sensors for protein amyloid aggregates. Dyes and Pigments. 2016. 132: 274-281. DOI: 10.1016/j.dyepig.2016.04.053

35. Kovalska V., Chernii S., Losytskyy M., Tretyakova I., Dovbii Y., Gorski A., Chernii V., Czerwieniec R., Yarmoluk S. Design of functionalized $\beta$-ketoenole derivatives as efficient fluorescent dyes for detection of amyloid fibrils. New Journal of Chemistry. 2018. 42 (16): 13308-13318. DOI: 10.1039/ c8nj01020j

36. Moshynets O., Chernii S., Chernii V., Losytskyy M., Karakhim S., Czerwieniec R., Pekhnyo V., Yarmoluk S., Kovalska V. Fluorescent $\beta$-ketoenole AmyGreen dye for visualization of amyloid components of bacterial biofilms. Methods and Applications in Fluorescence. 2020. 8 (3): 035006. DOI: $10.1088 / 2050-6120 / \mathrm{ab} 90 \mathrm{e} 0$

Стаття надійшла 17.06.2021. 